22. Nyhan WL, Fawcett N, Ando T, Rennert OM, Julius RL 1973 Response to dietary therapy in $B_{12}$ unresponsive methylmalonic acidemia. Pediatrics 51:539

23. Picou D, Phillips M 1972 Urea metabolism in malnourished and recovered children receiving a high or low protein diet. Am J Clin Nutr 25:1261

24. Pozefsky T, Marliss E, Cahill GL 1970 Alanine: key role in gluconeogenesis. Science 167:1003

25. Rose WC, Dekker 1956 Urea as a source of nitrogen for the biosynthesis of amino acids. J Biol Chem 181:107

26. Ruch T, Kerr D 1982 Decreased essential amino acid requirements without catabolism in phenylketonuria and maple syrup urine disease. Am J Clin
Nutr 35:217

27. Satoh $T$, Narisawa K, Igarashi $Y$, Saitoh $T$, Hayasaka K, Ichinohazama $Y$, Onodera H, Tada K, Oohara K 1981 Dietary therapy in two patients with vitamin $B_{12}$-unresponsive methylmalonic acidemia. Eur J Pediatr 135:406

28. Snell K 1980 Muscle alanine synthesis and hepatic gluconeogenesis. Biochem Soc Trans 8:205

29. Snyderman SE 1974 The amino acid requirements of the infant. In: Nyhan WL (ed) Heritable Disorders of Amino Acid Metabolism. John Wiley \& Sons, New York, pp 641-651

30. Snyderman SE, Holt LE, Dancis J, Roitman E, Boyer A, Balis ME 1962 "Unessential" nitrogen: a limiting factor for human growth. J Nutr 78:59

\title{
The Metabolic and Endocrine Milieu of the Human Fetus and Mother at 18-21 Weeks of Gestation. I. Plasma Amino Acid Concentrations
}

\author{
G. SOLTESZ,' D. HARRIS, I. Z. MACKENZIE, AND A. AYNSLEY-GREEN ${ }^{2}$ \\ University Department of Paediatrics, and Nuffield Department of Obstetrics and Gynaecology. John Radcliffe \\ Hospital, Oxford, England
}

\begin{abstract}
Plasma levels of 17 amino acids were measured in 10 conscious mothers and their minimally stressed fetuses (mean body weight $302 \mathrm{~g}$ ) at 18-21 wk gestation. Simultaneous blood samples were taken from the maternal antecubital vein, and from the fetal umbilical vein and artery by fetoscopy prior to termination of pregnancy. The mean concentrations of all amino acids were significantly higher in the fetal $(2.26 \mathrm{mmol} / \mathrm{liter})$ than in the maternal $(0.96 \mathrm{mmol} / \mathrm{liter})$ circulation, the total molar concentration of amino acids was 2.4 times greater in fetal than in maternal plasma. There was a significant positive relationship between maternal and fetal levels for most amino acids; the mean umbilical concentration difference was significantly positive for glycine, alanine, isoleucine, leucine, phenylalanine, and histidine. (Pediatr Res 19: 91-93, 1985)
\end{abstract}

\section{Abbreviations}
AA, amino acids
VA, venous-arterial
UA, umbilical artery
UV, umbilical vein
MV, maternal antecubital vein

The growing human fetus requires a continuous delivery of substrates and fuels, including $\mathrm{AA}$, for the synthesis of new

Received September 7, 1983; accepted August 1, 1984.

Requests for reprints should be addressed to Dr. G. Soltesz, Department of Paediatrics, University Medical School, Pecs, Hungary.

${ }^{1}$ Recipient of a Smith and Nephew Fellowship and Visiting Fellow of Green College, Oxford

2 James Spence Professor of Child Health, University of Newr" sile unon Tyne, England. tissue. It has been shown that in most mammals, including man, the umbilical circulation is the only important route by which the fetal body acquires substrates from the maternal environment (2).

Although the umbilical uptake of substrates by the fetus can be measured by the application of the Fick principle (in which umbilical blood flow and venous arterial concentration differences are determined simultaneously), to date, however, accurate measurement of the fetal uptake of the individual AA has been made in only one species, the fetal lamb, and then only late in gestation $(8,9)$.

No human data are available on uptake of AA and information on the circulating concentrations of AA during early gestation is scarce $(3,10,12)$. Moreover, the usefulness of the available data is limited because of the circumstances of blood collection. Thus, earlier studies were performed on fetuses at hysterotomy after placental separation and under maternal anaesthesia $(3,12)$, while only one fetal vessel was sampled in a more recent study in which blood samples were obtained by fetoscopy (10).

In order to overcome these difficulties we have taken advantage of the opportunity to collect simultaneous maternal and fetal artery and vein blood samples during fetoscopy without significant uterine or fetal manipulation. Herein we report results of measurement of plasma AA concentrations. Circulating concentrations of other metabolites, metabolic fuels, and hormones have also been measured and will be reported separately (1).

\section{PATIENTS AND METHODS}

Ten mothers at 18-21 wk gestation (mean $19 \mathrm{wk}$ ) were subjected to fetoscopy and fetal blood sampling immediately before termination of pregnancy for social reasons, within the terms of the 1967 Abortion Act; abortion was induced with intraamniotic instillation of prostaglandin immediately following the fetoscopic procedures. The study was approved by the hospital ethics committee and informed consent was obtained from the mothers. 
Table 1. Maternal and fetal plasma AA levels at 18-21 wk of gestation (mean \pm SEM)

\begin{tabular}{lrcccc}
\hline & MV & UA & UV & MV vs UA & MV vs UV \\
\hline Taurine & $22.4 \pm 1.7$ & $85.4 \pm 5.6$ & $81.8 \pm 3.7$ & $<0.001$ & $<0.001$ \\
Threonine & $115.2 \pm 7.7$ & $237.6 \pm 20.1$ & $258.7 \pm 18.2$ & $<0.001$ & $<0.001$ \\
Serine & $48.3 \pm 3.4$ & $85.5 \pm 7.1$ & $92.8 \pm 7.5$ & $<0.01$ & $<0.001$ \\
Glycine & $84.0 \pm 4.5$ & $126.5 \pm 9.3$ & $151.8 \pm 8.6$ & $<0.001$ & $<0.001$ \\
Alanine & $161.6 \pm 9.2$ & $225.8 \pm 13.6$ & $283.0 \pm 19.6$ & $<0.005$ & $<0.005$ \\
Citrulline & $16.5 \pm 1.5$ & $23.2 \pm 1.9$ & $25.4 \pm 1.8$ & $<0.05$ & $<0.005$ \\
$\alpha$-Amino isobutiric acid & $9.9 \pm 1.2$ & $23.4 \pm 2.2$ & $26.7 \pm 2.6$ & $<0.001$ & $<0.001$ \\
Valine & $122.3 \pm 7.2$ & $251.7 \pm 14.2$ & $277.2 \pm 14.2$ & $<0.01$ & $<0.005$ \\
Methionine & $10.5 \pm 0.8$ & $18.3 \pm 2.9$ & $19.8 \pm 2.5$ & $<0.001$ & $<0.001$ \\
Isoleucine & $36.4 \pm 2.6$ & $63.1 \pm 5$ & $72.9 \pm 4.1$ & $<0.001$ & $<0.001$ \\
Leucine & $66.1 \pm 3.9$ & $120.9 \pm 6.3$ & $137.8 \pm 6.1$ & $<0.001$ & $<0.001$ \\
Tyrosine & $23.0 \pm 1.6$ & $64.1 \pm 5.4$ & $64.6 \pm 4.4$ & $<0.001$ & $<0.001$ \\
Phenylalanine & $29.2 \pm 2.5$ & $55.9 \pm 4.3$ & $69.6 \pm 5.2$ & $<0.001$ & $<0.001$ \\
Ornithine & $19.7 \pm 1.1$ & $78.9 \pm 6.6$ & $82.6 \pm 6.6$ & $<0.001$ & $<0.001$ \\
Lysine & $106.2 \pm 7.1$ & $376.1 \pm 45$ & $418.2 \pm 33.5$ & $<0.001$ & $<0.001$ \\
Histidine & $54.5 \pm 3.6$ & $81.6 \pm 6.5$ & $96.4 \pm 5.4$ & $<0.005$ & $<0.001$ \\
Arginine & $30.2 \pm 1.9$ & $90.1 \pm 5.7$ & $99.5 \pm 6.5$ & $<0.001$ & $<0.001$ \\
\hline
\end{tabular}

The mothers fasted for $12-14 \mathrm{~h}$ prior to fetoscopy. They remained conscious during the procedure but were sedated $2 \mathrm{hr}$ preoperatively with $20 \mathrm{mg}$ papaveretum given intramuscularly, and then with $5 \mathrm{mg}$ diazepam given intravenously $10 \mathrm{~min}$ before the fetoscopy.

Blood samples of $1.0 \mathrm{ml}$ were withdrawn from the UA and UV under direct vision without physical manipulation or disturbance of the fetus: samples were confirmed to be of fetal origin by using the Kleihauer staining technique. Simultaneous MV blood samples were also collected.

Gestational age was determined from the date of the last menstrual period and confirmed by ultrasound. The mean body weight of the fetuses was $302 \mathrm{~g}$ (range 173-446 g).

Immediately on withdrawing, blood was added to a heparinized tube and centrifuged. Plasma AA were measured in the protein-free supernatant of plasma by automated ion-exchange chromatography by using ninhydrin detection. The interassay coefficient of variation for residues was $3.9 \%$.

Differences in means of maternal and fetal concentrations were tested by the unpaired Student's $t$ test, and umbilical VA differences were tested by the paired $t$ test. Linear regression equations were calculated by the least squares method, and their slopes were tested for significance by the $\mathrm{F}$ ratio test.

\section{RESULTS}

The concentrations of individual AA in MV and fetal vein and artery are compared in Table 1 . It is apparent that all AA had a significantly higher concentration in fetal than in maternal plasma. The fetal-maternal ratio, therefore, was greater than 1.0 for all AAs, but there was considerable variation with a range from 1.4-4. The total molar concentration of the 17 AA measured in this study was 2.4 times greater in fetal than in maternal plasma (2.26 versus $0.96 \mathrm{mmol} /$ liter).

There was a significant direct linear relationship between maternal and fetal levels for $14 \mathrm{AA}$ the slope of the regression line being similar for most AA (Table 2).

The mean umbilical VA concentration differences are ordered according to magnitude in Figure 1. It can be seen that the mean VA concentration difference was positive for the majority of AA. Plasma concentrations of glycine, alanine, isoleucine, leucine, phenylalanine, and histidine were significantly $(p<0.05)$ higher in the UV. Mean fetal arterial plasma urea concentrations was not significantly higher than the mean MV urea level $(2.65 \pm$ 0.77 versus $2.1 \pm 0.52 \mathrm{mmol} /$ liter, mean $\pm \mathrm{SEM}$ ) and there was no significant VA concentration difference for urea $(2.56 \pm 0.63$ versus $2.65 \pm 0.77 \mathrm{mmol} / \mathrm{liter}$, mean $\pm \mathrm{SEM}$ ).
Table 2. The relationship between maternal and fetal plasma amino acid levels; correlation coefficients ( $M V$ vs $F A$ )

\begin{tabular}{lcc}
\hline & $\mathrm{R}$ & $p$ \\
\hline Alanine & 0.66 & $<0.05$ \\
Valine & 0.80 & $<0.01$ \\
Threonine & 0.77 & $<0.01$ \\
Lysine & 0.86 & $<0.01$ \\
Glycine & 0.66 & $<0.05$ \\
Leucine & 0.71 & $<0.05$ \\
Histidine & 0.66 & $<0.05$ \\
Serine & 0.73 & $<0.05$ \\
Isoleucine & 0.77 & $<0.01$ \\
Arginine & 0.71 & $<0.05$ \\
Phenylalanine & 0.84 & $<0.01$ \\
Tyrosine & 0.73 & $<0.05$ \\
Ornithine & 0.71 & $<0.05$ \\
Methionine & 0.84 & $<0.01$ \\
\hline
\end{tabular}

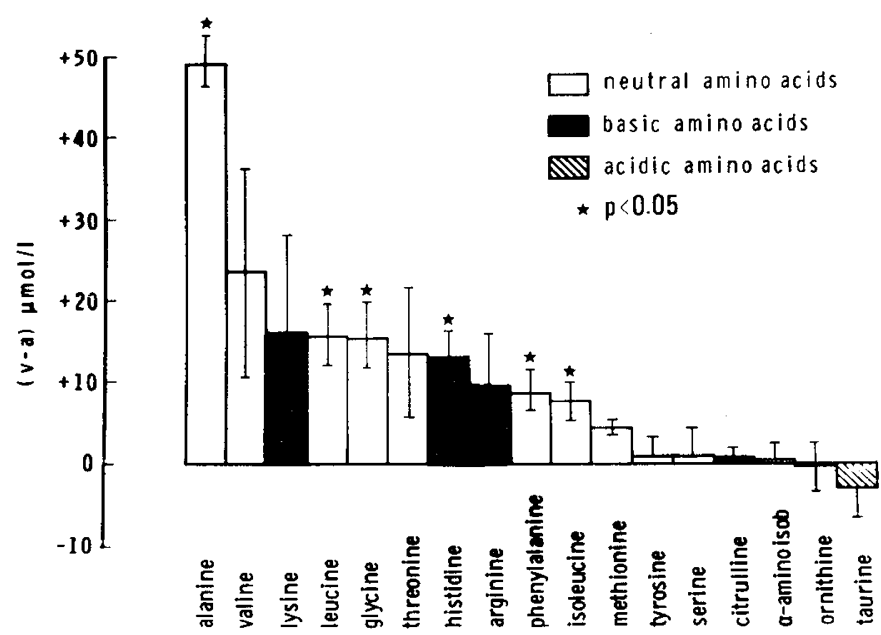

Fig. 1. Differences in UV and VA concentrations of plasma AA $(n=$ 10$, mean $\pm \mathrm{SEM})$.

\section{DISCUSSION}

The existence of a higher fetal than maternal $\alpha$-amino nitrogen concentration has been described previously in all species studied, including man during both early and late gestation (for review see Ref. 2). However, the validity of the observations for 
early human pregnancy made at hysterotomy has been questioned in a recent paper (10) because of the unphysiological conditions of sampling. The results of the present study confirm the existence of a fetomaternal gradient for AA at midterm gestation in human pregnancy. The total molar concentration of the $17 \mathrm{AA}$ measured in our study were more than twice as high in the fetal than in the maternal plasma.

Apart from confirming the presence of a fetomaternal gradient for AA, the present results show that there are important quantitative and qualitative differences between our data and those derived from hysterotomy studies. Fetal concentrations reported in these latter studies are generally much higher than those found in our study, probably due to the reduction in blood flow which occurs at hysterotomy through both the separated fetus and placenta (12). In fact, a similar rise, producing spuriously high fetal plasma AA levels have been also observed in the chronic sheep preparation during fetal surgery (12).

A further important observation questioning the physiological significance of data obtained at hysterotomy concerns the lack of a significant linear relationship between maternal and fetal plasma levels $(3,12)$. Our results are in agreement with previous reports from experimental animals (7) and human studies in late gestation (12) showing a significant positive correlation for most AA. This again suggests that during hysterotomy, impaired transfer occurs, most probably due to placental and fetal separation prior to sampling. It is also noteworthy that our data confirm substantial differences in the profiles of the concentrations of AA between the human and sheep fetus $(8,13)$.

The umbilical VA concentration differences found in the present study seem to confirm previous data obtained for the latter part of gestation $(6,8)$. However, net umbilical uptake of individual AA cannot be calculated from these measurements for the following reasons. First, significant positive AV difference (due to the large variation in the case of some AA, e.g. valine, lysine, threonine) was found for only six AA. Second, the umbilical VA difference for some AA (e.g. tyrosine, serine, ornithine) were smaller than the coefficient of variation for the method, thus exceeding the ability of chromatography to measure differences. Third, umbilical blood flow could not be measured.

Despite these limitations, our data are the first to describe umbilical venous and arterial plasma levels in the minimally stressed human fetus at midterm gestation. Furthermore, the demonstration of the largest significant positive VA concentration difference for alanine implies that there is a high uptake of this nonessential AA, making it the major source of fetal nitrogen as well as being possibly an important source of nonglucose carbon supplied by the mother $(6,11)$. This appears to be in contrast to the fetal lamb, where the greatest umbilical VA difference was found for glutamine (8). While emphasizing these important species differences, it is of interest to note that both alanine and glutamine are major substrates for transamination and gluconeogenesis. Nontheless it is clear that it is not possible to extrapolate data derived from the sheep fetus to man, particularly since sheep have a more rapid growth rate and an entirely different body composition (2).

Although the fetomaternal gradient for plasma urea was comparable to that found at term (5), the value did not achieve statistical significance ofwing to the wide scatter of the data. In agreement with earlier studies (2), no significant VA concentration difference could be detected for urea, making the application of the Fick principle impractical for the measurement of fetal urea excretion (4).

We conclude that blood samples derived from the fetus at fetoscopy allow a new approach to defining the interrelations of maternal and fetal metabolism during the 2nd trimester of human pregnancy. With the measurement of umbilical blood flow and further refinement of analytical techniques, it would be possible to quantify the nutrient requirements during gestation in man.

Acknowledgments. The authors thank Professor P. J. Randle for allowing GS to use his amino acid analyzer; Professor M. Young, Professor Randle, and Dr. Peter Lloyd for their advice and helpful criticism.

\section{REFERENCES}

1. Aynsley-Green A, Soltesz G, Jenkins PA, Mackenzie IZ, Adrian TE, Bloom SR 1985 The metabolic and endocrine milieu of the human fetus and mothe at 18-21 weeks of gestation. II. Intermediary Metabolite and metabolic hormone concentrations. Biol Neonate, in press

2. Battaglia FC, Meschia G 1978 Principal substrates of fetal metabolism. Physiol Rev 58:499

3. Cockburn F, Robins SP, Forfar JO 1970 Free amino acid concentrations in fetal fluids. Br Med J 2:747

4. Gresham EL, James EJ, Raye JR, Battaglia FC, Makowski EL, Meschia G 1972 Production and excretion of urea by the fetal lamb. Pediatrics $50: 372$

5. Gresham EL, Simons PS, Battaglia FC 1971 Maternal-fetal urea concentration differences in man: metabolic significance. J Pediatr 78:805

6. Hayashi S, Sanada K, Sagawa N, Yamada N, Kido K 1978 Umbilical veinartery differences of plasma amino acids in the last trimester of human pregnancy. Biol Neonate 34:11

7. Kerr GR, Chamore AS, Harlow HF, Waisman HA 1968 "Fetal PKU." The effect of maternal hyperphenylalaninaemia during pregnancy in the rhesus monkey (Macaca mulatta). Pediatrics 42:27

8. Lemons JA, Adcock EW, Douglas Jones M, Naughton MA, Meschia G Battaglia FC 1976 Umbilical uptake of amino acids in the unstressed fetal lamb. J Clin Invest 58:1428

9. Lemons JA, Schreiner RL 1983 Amino acid metabolism in the ovine fetus Am J Physiol 244:E459

10. McIntosh N, Rodeck CH, Heath R 1984 Plasma amino acids of the mid trimester human fetus. Biol Neonate 45:218-224

11. Young M Amino acid uptake by the fetus. Placenta 125(suppl 11):

12. Young M, Prenton MA 1969 Maternal and fetal plasma amino acid concentrations during gestation and in retarded fetal growth. J Obstet Gynecol $\mathrm{Br}$ Commonw 76:333

13. Young M, Soltesz G, Noakes D, Joyce J, McFadyen IR, Lewis BV 1973 The influence of intrauterine surgery and of fetal i.v. nutritional supplements "in-utero" on plasma free amino acid homeostasis in the pregnant ewe. J Perinat Med 3:180 